\title{
A $2 \mathrm{~m} \times 0.5 \mathrm{~m}$ prototype of a MRPC-based neutron detector with steel converter plates
}

\author{
Marko Röder $^{* a b}$, T. Aumann ${ }^{c d}$, D. Bemmerer ${ }^{b}$, K. Boretzky $^{c}$, C. Caesar ${ }^{c}$, T. E. \\ Cowan $^{a b}$, J. Hehner ${ }^{c}$, M. Heil' ${ }^{c}$, Z. Elekes ${ }^{b}$, M. Kempe ${ }^{b}$, V. Maroussov' ${ }^{e}$, T. P. \\ Reinhardt $^{a b}$, H. Simon ${ }^{c}$, M. Sobiella ${ }^{b}$, D. Stach ${ }^{b}$, A. Wagner ${ }^{b}$, J. Wüstenfeld ${ }^{b}$, D. \\ Yakorev $^{b}$, A. Zilges ${ }^{e}$, K. Zuber $^{a}$, for the $\mathbf{R}^{3}$ B Collaboration \\ ${ }^{a}$ Institut für Kern- und Teilchenphysik, Technische Universität Dresden, Germany \\ ${ }^{b}$ Institut für Strahlenphysik, Helmholtz-Zentrum Dresden-Rossendorf, Germany \\ ${ }^{c}$ GSI Helmholtzzentrum für Schwerionenforschung, Darmstadt, Germany \\ ${ }^{d}$ Technische Universität Darmstadt, Darmstadt, Germany \\ e Universität zu Köln, Köln, Germany
}

E-mail: Marke.Reederdu-dresden.de

\begin{abstract}
A $2 \mathrm{~m}$ long prototype detector for the detection of neutrons in an energy range from $200 \mathrm{MeV}$ to $1 \mathrm{GeV}$ has been successfully realized. The working principle is based on steel converter plates followed by an MRPC structure to detect charged particles produced by hadronic interactions. In order to study time resolution and efficiency, a $2 \mathrm{~m} \times 0.5 \mathrm{~m}$ large module has been built which comprises a $2 \times 2$ gap MRPC structure. An efficiency larger than $90 \%$ and a time resolution better than $100 \mathrm{ps}$ have been measured for minimum ionizing electrons. This experiment has been done using the one-electron-per-bunch mode of the superconducting electron linear accelerator ELBE, Dresden. Another test has been done using $175 \mathrm{MeV}$ quasi-monochromatic neutrons at TSL in Uppsala. A test using tagged high-energy neutrons is scheduled at GSI. Extensive Monte Carlo simulations have been carried out, both for the electron-beam tests and for the final application as a neutron detector. The present approach offers a cost-effective way for the time-of-flight detection of high energy neutrons.
\end{abstract}

XI workshop on Resistive Plate Chambers and Related Detectors - RCP2012,

February 5-10, 2012

INFN Laboratori Nazionali di Frascati Italy

\footnotetext{
* Speaker.
} 


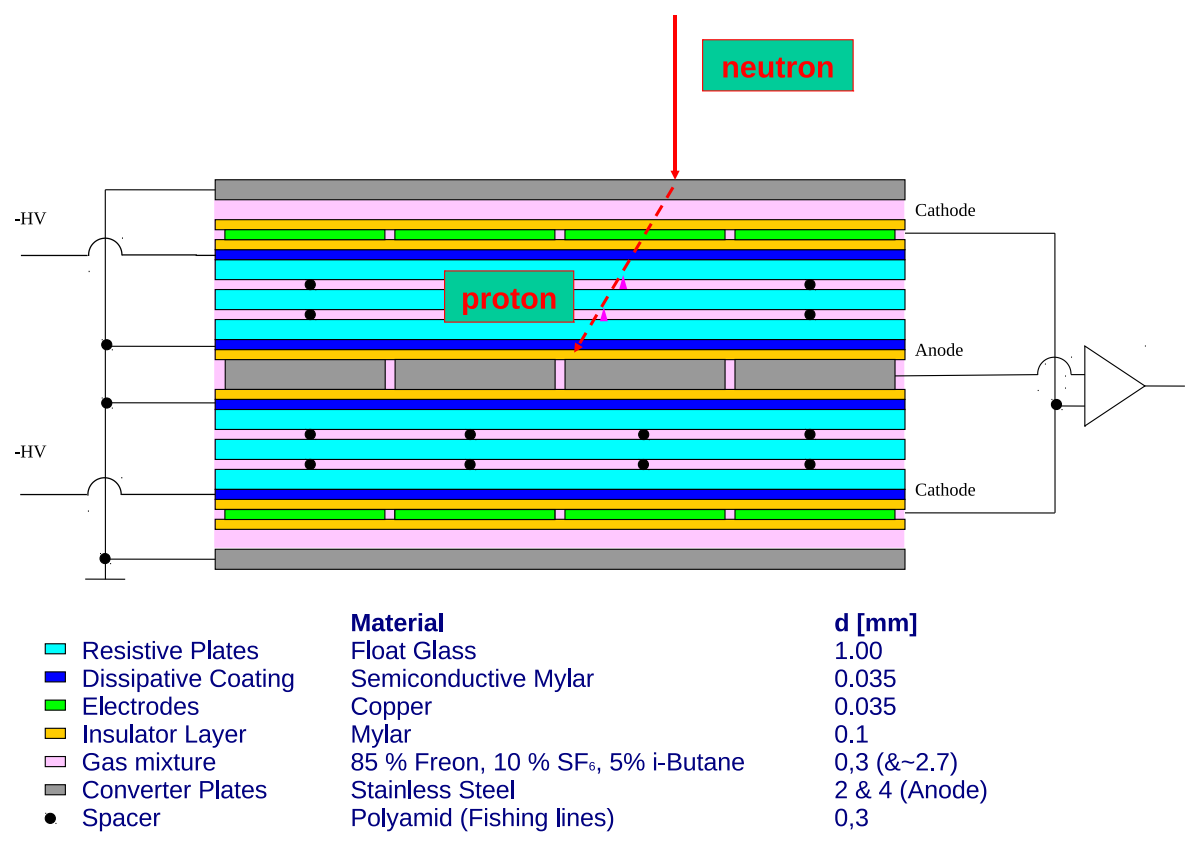

Figure 1: Schematic side view of the $2 \mathrm{~m} \times 0.5 \mathrm{~m}$ large prototype (not to scale).

\section{Introduction}

From 2007 on we developed an MRPC-based neutron detector with steel converter plates in the framework of the $\mathrm{R}^{3} \mathrm{~B}$ Collaboration (Reactions with Relativistic Radioactive Beams) [U]. Originally this detector concept was one option for the NeuLAND detector (New Large Area Neutron Detector) [3] at the future FAIR facility (Facility for Antiproton and Ion Research) at GSI, Darmstadt, Germany. The design goals for the NeuLAND detector are: detection of high energy neutrons in a range from 0.2 to $1 \mathrm{GeV}$, a time resolution $\sigma_{t} \leq 100 \mathrm{ps}$, an efficiency for $400 \mathrm{MeV}$ neutrons of $>90 \%$, with multi-neutron capabilities and an active area of $2 \mathrm{~m} \times 0.5 \mathrm{~m}$ in the full acceptance mode.

This development was done in several steps. In a first step we built small prototypes with an area of $40 \mathrm{~cm} \times 20 \mathrm{~cm}$ and we tested them using minimum ionizing electrons to study their properties and to extract the best design parameters to reach the design goals for the large modules with an area of $2 \mathrm{~m} \times 0.5 \mathrm{~m}$. The second step was to test these small prototypes with $175 \mathrm{MeV}$ quasi monochromatic neutrons and the last approach was to develop and test $2 \mathrm{~m} \times 0.5 \mathrm{~m}$ large modules with $30 \mathrm{MeV}$ electrons at the ELBE facility (Electron LINAC with high Brilliance and low Emittance) [ [ $]$ at Helmholtz-Zentrum Dresden-Rossendorf. While the first step is discussed in detail in ref. [ए]] this paper will concentrate on steps two and three.

\section{Adopted design for the $2 \mathrm{~m} \times 0.5 \mathrm{~m}$ prototype and detection principle}

From the tests of the small prototypes with minimum ionizing electrons (discussed in detail in [ए]]) and quasi monochromatic neutrons (discussed in section B]) we drew the following conclu- 


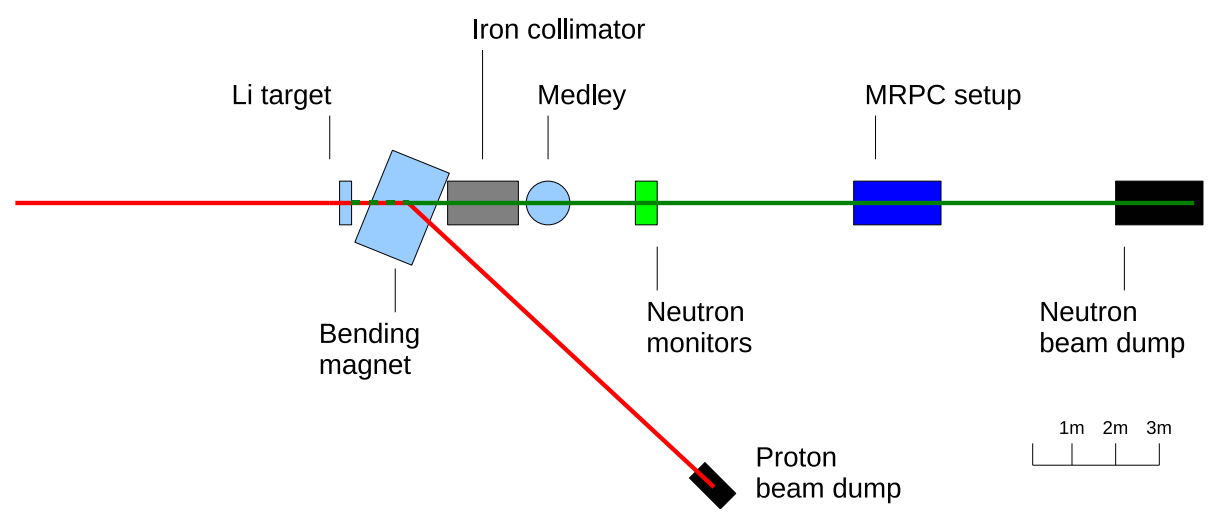

Figure 2: Schematic overview of the beamline in the Blue Hall of the TSL neutron facility.

sions for the design of the large prototype: a $2 \times 2$ gas gap structure is sufficient to reach the goals of $\eta>90 \%$ and $\sigma_{t}<100 \mathrm{ps}$, differential and single ended readout is possible, inter strip spacing $=1.5 \mathrm{~mm}$ to minimize crosstalk.

Impinging neutrons convert into charged particles, mainly protons, by quasi-elastic scattering and hadronic interactions. The charged particle produces electron avalanches in the amplifying gas while inducing signals at the readout electrodes. Signals are read out using dedicated Front End Electronics (FEE). A schematic view of the detector assembly and the detection principle can be seen in fig प.

\section{Detector tests of small prototypes with $175 \mathrm{MeV}$ neutrons at TSL in Uppsala}

In November 2009 we performed tests of the $40 \mathrm{~cm} \times 20 \mathrm{~cm}$ prototypes with $175 \mathrm{MeV}$ quasimonochromatic neutrons at The Svedberg Laboratory (TSL) in Uppsala [Q]. In fig. \ there is a schematic view of the beam facility.

The TSL cyclotron accelerates protons to $179.3( \pm 0.8) \mathrm{MeV}$. These protons interact with a ${ }^{7} \mathrm{Li}$ target producing neutrons of $175( \pm 2.5) \mathrm{MeV}$ and a low energy tail via a ${ }^{7} \mathrm{Li}(\mathrm{p}, \mathrm{n}){ }^{7} \mathrm{Be}$ reaction. From there the protons are bend away into a beam dump and the neutrons are shaped by a cylindrical iron collimator. Afterwards the neutrons move through the Medley spectrometer [[]] and impinge onto the MRPC-Detector-Setup.

Two neutron monitors, namely a Thin Film Breakdown Counter (TFBC) and an Ionization Chamber Monitor (ICM), were located in between the Medley and the MRPC setup in order to infer the number of incoming neutrons on the MRPC setup. Downstream of the proton-branch there also was a proton monitor in the Beam Dump.

From the tests we could extract the TOF- and the energy spectrum of our detector units. Figure (3(a) shows the micro structure of the TSL neutron beam with its repetition period of $45 \mathrm{~ns}$ measured with our MRPCs, normalized to total number of counts. Within the $200 \mathrm{~ns}$ time-to-digital window we could cover four of these micro pulses. In figure $[3(\mathrm{~b})$ the TOF is directly converted into the energy spectrum (red line) and normalized. In addition we show a reference spectrum measured by the Medley spectrometer [[]] (renormalized). 

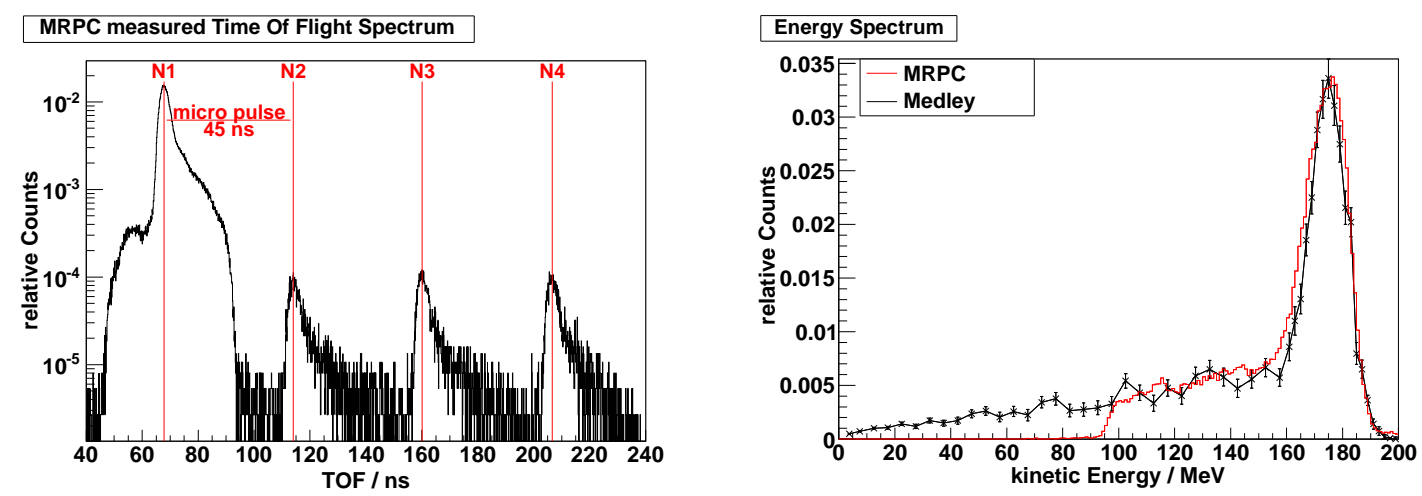

Figure 3: Normalized spectra measured with the MRPC at TSL in Uppsala. Left panel (a): TOF spectrum, representing the micro structure of the TSL cyclotron. Right panel (b): Energy spectrum, converted from the measured TOF spectrum. Medley spectrum taken from [ㅁ] and renormalized.

\section{Detector tests at ELBE}

After constructing the $2 \mathrm{~m} \times 0.5 \mathrm{~m}$ large prototypes we tested them at the ELBE facility (Electron LINAC with high Brilliance and low Emittance) at Helmholtz-Zentrum Dresden-Rossendorf. The facility provides electrons with $30 \mathrm{MeV}$ kinetic energy, close to the minimum of ionization. Furthermore it can be operated in the new single-electron-per-bunch mode where the beam is thinned out using Al-scattering-foils such that each bunch contains only one electron [ [ $]$ ]. The RF signal of the ELBE accelerator was used as time reference. It was logically combined with two scintillators in front of our detector. The resulting signal was used as trigger and fed back again into a readout channel measuring a $\sigma_{t, R F} \leq 35 \mathrm{ps}$ (including jitter of RF and readout electronics).

Beside the usual electronics that was discussed in [0] we also used the new TacQuila-Electronics [ [ $]$ ] developed at GSI. In our experiments an extended version of the electronics called TacQuila ${ }^{17}$ was used. In addition to 16 signal channels this extended version is providing an additional channel (channel 17) working as a common stop signal [四]. The analysis procedure for the TacQuila readout was similar to the one described in [ए]]. An efficiency- and time resolution curve as function of the electric field strength can be seen in fig. H(a). Prototype HZDR201b was tested at the center of strip 4 with a trigger rate of $350 \mathrm{~Hz}$ for different high voltages. Variations of the detection efficiency and the time resolution on the position along different strips with the usual readout can be seen in fig. 4(b).

\section{Simulation for fast neutrons}

Monte Carlo simulations have been performed using the Geant 4 toolkit to study the response of the detector for fast neutrons and to reconstruct multi neutron events (discussed in detail in [[]]). All simulations have been done using a Monte Carlo engine that provides the primary neutrons and their physical properties. After that the avalanche growth (governed by the Townsend and attachment coefficients) inside the gas gaps of the MRPCs and the signal formation was simulated.

Two consecutive simulations have been carried out. First the $40 \mathrm{~cm} \times 20 \mathrm{~cm}$ prototypes were coded and major calibration parameters were extracted by comparing simulated results to experi- 

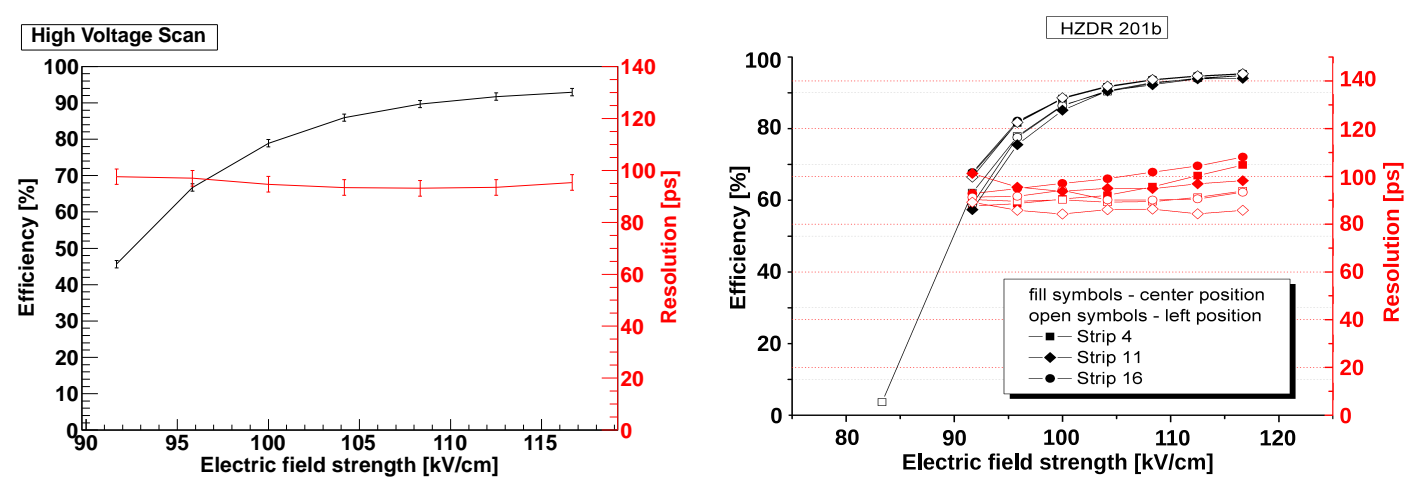

Figure 4: Efficiency and time resolution measured with HZDR201b. Left panel (a): data measured with TacQuila electronics at the center of strip four as function of electric field strength. Right panel (b): data measured with usual electronics at the center and the edge of several strips

mental data with minimum ionizing electrons from ref. [ए0]. Especially the space charge effect, the interplay of avalanches (important for signal merging) and a threshold for the final electronic-signal were extracted. Figure 5(a) shows such a comparison of experimental with simulated data.

The second simulation was used to study the response of the large prototype to high energy neutrons. The large prototypes were coded and the extracted calibration parameters from the small prototypes were applied. Furthermore a random noise and a time resolution of $\sigma_{t}=150 \mathrm{ps}$ (as worst case scenario) were implemented. Hypothetic event files were used providing neutrons from the reaction ${ }^{132} \mathrm{Sn} \rightarrow{ }^{(132-\mathrm{x})} \mathrm{Sn}+\mathrm{xn}(\mathrm{x}=1,2,3,4)$ at 200,600 and $1000 \mathrm{MeV} /$ nucleon.

From these studies we conclude that the design goal of $\eta>90 \%$ for $400 \mathrm{MeV}$ neutrons can be reached with 50 layers of MRPC structures leading to a total depth of $1.2 \mathrm{~m}$.

In order to determine the neutron momenta for multi neutron events, an algorithm is necessary that can reconstruct the number of incident primary neutrons [5]. Hits within a certain distance are combined into clusters, the velocity between each cluster is calculated and clusters with a velocity smaller than the beam velocity are excluded. The number of the surviving clusters give the number of infalling primary neutrons. Also the multiplicity of one event is taken into account [1]. Using the surviving hits after the reconstruction algorithm, we can calculate the momenta and the energy of the neutrons and thus reconstruct the relative energy spectrum. In fig. 5(b) such reconstructed relative energy spectra for a $1 \mathrm{n}$ case is shown. Based on the reconstruction algorithm we could extract a resolution $\sigma_{E r e l}=17 \mathrm{keV}$ for the $1 \mathrm{n}$ case. The multineutron response of the array is under investigation.

\section{Summary and outlook}

In this paper we showed that MRPCs with steel converter plates can be used to detect neutrons in an energy range from 0.2 to $1 \mathrm{GeV}$. We performed efficiency and time resolution tests using minimum ionizing electrons from the superconducting linear accelerator ELBE at HZDR reaching efficiencies $\eta>90 \%$ and time resolutions $\sigma_{t}<100 \mathrm{ps}$. Simulations showed high detection efficiency of the full setup for primary neutrons. 

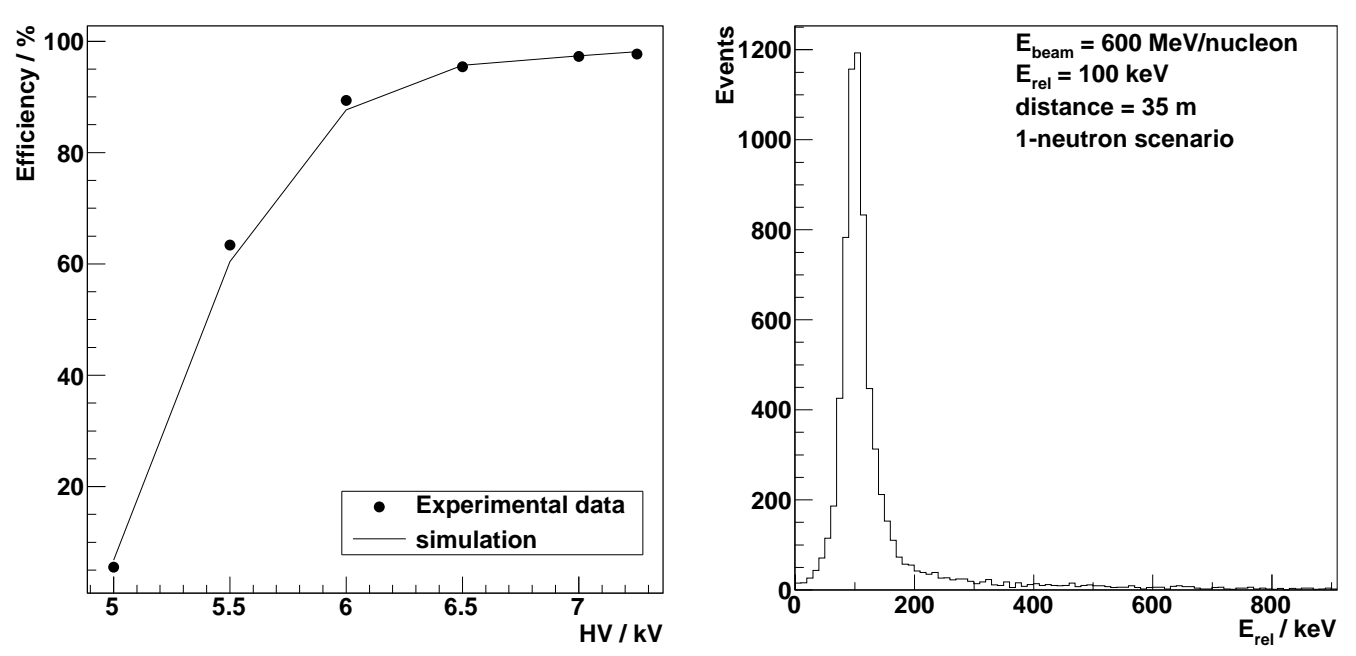

Figure 5: Left panel (a): Efficiency as function of the High Voltage, comparison of simulated (solid line) with experimental data (dots) for a $2 \times 2$ gap prototype. Right panel (b): Reconstructed relative energy spectrum for a 1 n scenario.

In the future further analysis of the $175 \mathrm{MeV}$ neutron beam data measured with the small prototypes is ongoing. A test of the large prototypes with fast neutrons from deuteron breakup is planned for 2012 at GSI, Darmstadt. The $R^{3} B$ collaboration decided to opt for a scintillatorbased solution for the NeuLAND detector at FAIR because of its better multi neutron capabilities. Therefore the presently described detector is open for collaboration.

\section{Acknowledgments}

This work was supported in part by GSI F\&E (DR-ZUBE), BMBF(06DR134I,06KY9136), NupNET NEDENSAA (BMBF 06DR05NUP) and the European Union (FP6-EFNUDAT).

\section{References}

[1] T. Aumann, et al., Prospects of nuclear structure at the future GSI accelerators, 2007, Prog. Part. Nucl. Phys. 59, p. 3

[2] R. Bevilacqua, et al., Medley spectrometer for light ions in neutron-induced reactions at $175 \mathrm{MeV}$, 2011, Nucl. Instr. Meth. A 646, p. 100

[3] K. Boretzky, et al., NeuLAND Technical Design Report, 2011, submitted to FAIR

[4] C. Caesar, et al., New LAND electronics - TacQuila17 - Version 0.8, TacQuila-manual, GSI, private communication, July 2011

[5] Z. Elekes, et al., Simulation and prototyping of $2 \mathrm{~m}$ long resistive plate chambers for detection of fast neutrons and multi-neutron event identification, 2012, to be submitted to Nucl. Instr. Meth. A

[6] F. Gabriel, et al., The Rossendorf radiation source ELBE and its FEL projects, 2000, Nucl. Instr. Meth. B 161-163, p. 1143 
[7] K. Koch, et al., A New TAC Based Multi Channel Front-End Electronics for TOF Experiments with Very High Time Resolution, 2005, IEEE TRANSACTIONS ON NUCLEAR SCIENCE 52 (3), p. 745

[8] L. Naumann, et al., Anordnung und Verfahren zur Erzeugung einzelner relativistischer Elektronen, 2008, Patent DE 102008054676Al

[9] A. V. Prokofiev, et al., The TSL neutron beam facility, 2007, Radiation Protection Dosimetry 126, p. 18

[10] D. Yakorev, et al., Prototyping and tests for an MRPC-based time-of-flight detector for $1 \mathrm{GeV}$ neutrons, 2011, Nucl. Instr. Meth. A 654, p. 79 